Nervenarzt $2008 \cdot 79: 577-586$

DOI 10.1007/s00115-008-2467-5

Online publiziert: 25 . April 2008

(c) Springer Medizin Verlag 2008

A. Maercker ${ }^{1}$ S. Forstmeier ${ }^{1}$ B. Wagner ${ }^{1} \cdot$ H. Glaesmer ${ }^{2} \cdot$ E. Brähler ${ }^{2}$

${ }^{1}$ Fachrichtung Psychopathologie und Klinische Intervention, Universität Zürich

${ }^{2}$ Abteilung für Medizinische Psychologie und Medizinische

Soziologie, Universitätsklinikum Leipzig, Leipzig

\title{
Posttraumatische Belastungsstörungen in Deutschland
}

\section{Ergebnisse einer gesamtdeutschen epidemiologischen Untersuchung}

Die posttraumatische Belastungsstörung (PTBS) ist eine schwere und oft chronische Störung bei einem Teil der Menschen, die extrem belastenden bzw. traumatisierenden Ereignissen ausgesetzt waren. Die meisten epidemiologischen Befunde zur PTBS stammen aus den USA, wo unter Benutzung von DSM-III-R- oder DSM-IVKriterien in mehreren Studien Lebenszeitprävalenzen von $5-10 \%$ berichtet wurden $[1,2]$.

In Deutschland gab es bislang nur epidemiologische Studien mit repräsentativen Stichproben von Jugendlichen oder jungen Erwachsenen, die eine Lebenszeitprävalenz von $1,3 \%$ bei 14 - bis 24 -jährigen Frauen und Männern [3] und 3,2\% bei 18bis 45-jährigen Frauen [4] fanden. Jüngst hat eine Studie an 18- bis 64-Jährigen eine Lebenszeitprävalenz von 1,4\% sowie eine 12 -Monats-Prävalenz von $0,7 \%$ berichtet [5].

Relativ niedrige Lebenszeitprävalenzraten in Höhe von 1,4\% fand die „European Study of the Epidemiology of Mental Disorders (ESEMeD) " [6], die verschiedene Länder (u. a. Deutschland, Spanien, Frankreich, Belgien) abdeckt. Aus einigen weiteren Ländern (Mexiko, Australien, Portugal) liegen repräsentative Bevölkerungsdaten vor, deren Prävalenzschät- zungen ebenfalls alle geringer sind als die der US-Studien. In Mexiko fand man bei 18- bis 65-Jährigen eine PTBS-Lebenszeitprävalenz von 1,4\% [7] und in Australien bei der gleichen Altersgruppe eine 12Monats-Prävalenz von 1,3\% [8]. Dagegen wurden in Portugal bei 18- bis 99-Jährigen eine Punktprävalenz von 5,3\% sowie eine Lebenszeitprävalenz von $7,9 \%$ gefunden, die hauptsächlich mit der hohen Anzahl von älteren Probanden, die in den Kolonialkriegen der 196oer und 1970er Jahre beteiligt waren, erklärt wurden [9].

Für die Erklärung der Prävalenzunterschiede zwischen verschiedenen Ländern kann man neben Naturkatastrophen auch den Einfluss von Kriegsgeschehen und der allgemeinen Gewalthäufigkeit in der Gesellschaft heranziehen [10]. In Deutschland sind die Teilnehmer und Überlebenden des 2. Weltkriegs in der älteren Bevölkerung stark vertreten. Es ist von daher zu erwarten, dass bei den über 6o-Jährigen in Deutschland sowohl hohe Raten kriegsbedingter Traumatisierungen als auch erhöhte PTBS-Prävalenzraten vorhanden sind. Epidemiologische Studien in der Allgemeinbevölkerung, die insbesondere die über 6o-Jährigen mitberücksichtigen, liegen bisher jedoch noch nicht vor.

Um eine größtmögliche Abdeckung der Altersspanne (u. a. in Hinblick auf die Kriegserlebnisse der Älteren) zu gewähr- leisten, wurden für die vorliegende Studie eine repräsentative Stichprobe von 14bis 95-Jährigen genutzt. Dabei musste allerdings die Beschränkung in Kauf genommen werden, nur die Ein-MonatsPTBS-Prävalenz zu erfassen, da die Repräsentativstudie nur einen begrenzten Umfang der erhobenen Datenmenge erlaubte. Die Ziele der Studie waren, zuverlässige Angaben über die Prävalenz von traumatischen Ereignissen und posttraumatischen Belastungsstörungen zu erhalten sowie bedingte Wahrscheinlichkeiten für die PTBS-Entstehung in Folge spezifischer Traumatatypen zu untersuchen. Insbesondere interessierten uns hierbei auch Altersgruppenunterschiede und inwiefern traumatische Erfahrungen im 2. Weltkrieg noch einen entsprechenden Stellenwert bei den berichteten Traumata hatten.

\section{Methoden}

\section{Stichprobe}

Die Untersuchung wurde im Sommer 2005 mit Hilfe eines Umfrageinstituts (USUMA, Berlin) mittels Befragung durch geschulte Mitarbeiter durchgeführt. Das Stichprobenverfahren basierte auf der „Random-route-mit-Startadressen-Methode“, bei der computergestützt zufällig 


\begin{tabular}{|lll}
\hline Tab. 1 Soziodemographische Merkmale ( $\mathbf{n}=\mathbf{2 4 2 6 )}$ & \\
\hline Merkmale & Anzahl [n] & \\
\hline Geschlecht & & \\
\hline Weiblich & 1118 & 46,08 \\
Männlich & 1308 & 53,92 \\
\hline Alter [Jahre] & & \\
\hline 14-29 & 382 & 15,75 \\
30-59 & 1230 & 50,70 \\
60-93 & 814 & 33,55 \\
\hline Familienstand/Partnerstatus & & \\
Verheiratet & 1353 & 55,77 \\
Ledig & 534 & 22,01 \\
Geschieden & 226 & 9,32 \\
Verwitwet & 313 & 12,90 \\
Zusammenlebend & 1475 & 60,80 \\
Nicht zusammenlebend & 951 & 39,20 \\
\hline Bildungsstand & & \\
\hline Bis Haupt-/Volksschulabschluss & 1202 & 49,54 \\
Mittlere Reife/Realschule/POS & 914 & 37,67 \\
Fach-/Hochschulreife od. -studium & 310 & 12,78 \\
\hline Erwerbstätigkeit & & \\
In Ausbildung & 160 & 6,60 \\
Voll- oder Teilzeiterwerbstätigkeit & 1109 & 45,71 \\
Nicht berufstätig & 219 & 9,03 \\
Arbeitslos & 181 & 7,46 \\
Renter/Pensionäre/Vorruhestand & 757 & \\
\hline Wohnsitz & 499 & \\
\hline Ostdeutschland (Neue Bundesländer) & 1927 & \\
Westdeutschland (Alte Bundesländer) & & \\
\hline
\end{tabular}

Haushalte generiert werden, deren Telefonnummern als Ausgangsdaten genutzt wurden. Insgesamt wurden zu 258 Rekrutierungspunkten in ganz Deutschland Adressen generiert (alte Bundesländer: 210, neue Bundesländer: 48), um eine möglichst große regionale Repräsentanz $\mathrm{zu}$ erreichen.

Innerhalb des zufällig ausgesuchten Haushalts wurde die Zielperson nach der "Last-birthday-Methode“ bestimmt. Die Datenerhebung erfolgte durch Vorlage von Fragebögen im Beisein der Umfrageinstitutsmitarbeiter. Zur Herstellung des Erstkontakts mit einem Haushalt und der Zielperson wurden jeweils bis zu 6 Anrufe getätigt. Die ethischen Leitlinien des Internationalen Kodex für die Praxis von Sozial- und Marktforschung der Europäischen Gesellschaft für Meinungs- und Marketingforschung wurden eingehalten.

Entsprechend diesem Verfahren wurden 4118 Privatpersonen aus allen Bundesländern für die Studie ausgewählt. 1018 $(25,7 \%)$ der angefragten Personen waren nicht zu erreichen. $428(10,4 \%)$ lehnten die Teilnahme am Interview ab; über die Zusage oder Absage der Teilnahme wur-

\section{Instrumente}

Die Auswahl der Befragungsinstrumente wurde nach Maßgabe ihrer Validität und Kürze zusammengestellt bzw. aufgrund eigener Vorarbeiten modifiziert.

\section{Liste mit traumatischen Ereignissen}

Entsprechend einer frühen Version der Traumaliste des PTSD-Moduls (vgl. [12]) des Münchener Composite International Diagnostic Interviews (M-CIDI [13]) wurden 8 potenziell traumatisierende Ereignisse vorgegeben (z. B. „Sie waren Opfer: einer Vergewaltigung “; ,.... einer Naturkatastrophe“; „Sie wurden ernsthaft körperlich bedroht [z. B. mit einer Waffe], angegriffen, verletzt oder gequält“; „Sie haben bei einem Kriegseinsatz schreckliche Erfahrungen gemacht") sowie eine offene Frage über „ein anderes schreckliches Ereignis oder eine Katastrophe“. Zusätzlich wurden nach 3 weiteren Ereignissen gefragt („Sie wurden ausgebombt“; „Sie wurden heimatvertrieben“; „Bei ihnen wurde eine lebensbedrohliche Krankheit diagnostiziert"), zu denen die Teilnehmer Angaben machen konnten. Darauf folgten Fragen nach dem DSM-IV-A2-Kriterium (intensive Furcht, Entsetzen und Hilflosigkeit).

Auf diese Angaben folgte die Nachfrage nach dem am meisten belastenden Ereignis (falls mehrere Ereignisse angegeben wurden). Wenn die Teilnehmer mehrere Ereignisse angegeben hatten, bezogen sich die nachfolgende Frage sowie die Symptomerfassung auf dieses am meisten belastende Ereignis.

\section{Modifizierte PTSD-Symptomskala}

Die DSM-IV-Symptomkriterien wurden im Format der Posttraumatic Symptom Scale (PSS [14]) erfragt. Die Antworten bezogen sich auf das Vorkommen im letzten Monat mit einer 4-stufigen Antwortskala von o (,überhaupt nicht"), 1 („,einmal pro Woche oder seltener"), 2 („2- bis 4-mal pro Woche/die Hälfte der Zeit") bis 3 ("mehrfach pro Woche/fast immer"). Einige der PTSD-Symptomkriterien, die nach Breslau et al. [15] eine geringe diagnostische Prädiktion (Sensibilität, Sensitivität) zeigen, wurden aufgrund des Screeningcharakters der Studie weggelassen (Kriterien $\mathrm{B}_{3}, \mathrm{~B}_{4}, \mathrm{C}_{2}, \mathrm{C}_{3}, \mathrm{D}_{2}-\mathrm{D}_{4}$ ). Das F-Kriterium (allgemeines DSM-IV- 
Hier steht eine Anzeige.

算 Springer 
Nervenarzt 2008 · 79:577-586 DOI 10.1007/s00115-008-2467-5

(c) Springer Medizin Verlag 2008

\section{A. Maercker · S. Forstmeier · B. Wagner · H. Glaesmer · E. Brähler Posttraumatische Belastungsstörungen in Deutschland. Ergebnisse einer gesamtdeutschen epidemiologischen Untersuchung}

\section{Zusammenfassung}

In einer repräsentativen Bevölkerungsstichprobe $(n=2426)$ über ein breites Alterspektrum (14-93 Jahre) wurden die Prävalenz traumatischer Ereignisse, des Vollbildes der posttraumatische Belastungsstörung (PTBS) und partieller PTBS-Syndrome geschätzt. Ein standardisiertes Interview mit einer Traumaliste des Composite International Diagnostic Interviews (CIDI) und eine PTSD-Symptomliste nach DSM-IV (modifizierte PTSD-Symptomskala) wurden eingesetzt. Die Einmonatsprävalenzrate lag bei $2,3 \%$ für das PTBS-Vollbild sowie 2,7\% für die partiellen PTBS-Syndrome. Während sich keine Geschlechtsunterschiede hinsichtlich der Prävalenz ergaben, zeigten sich Altersgruppenunterschiede: Die über 60Jährigen hatten eine Prävalenz bez. des PTBSVollbildes von 3,4\%, während diese bei den 14- bis 29 -Jährigen $1,3 \%$ und bei den 30 - bis
59-Jährigen 1,9\% betrug. Die partiellen PTBSSyndrome zeigten ebenfalls einen Altersgipfel mit 3,8\% in der Gruppe der Älteren sowie $2,4 \%$ bei Mittelalten und $1,3 \%$ bei jungen Erwachsenen. Die Ergebnisse entsprechen weitgehend denen anderer internationaler Studien, wenn Kriegsauswirkungen für bestimmte Altersgruppen als nationale Besonderheiten berücksichtigt werden. Erstmals konnte unsere repräsentative Studie eine wahrscheinlich durch den 2 . Weltkrieg mitbedingte - relativ hohe Prävalenz der PTBS in der höchsten Altersgruppe der deutschen Bevölkerung aufzeigen.

Schlüsselwörter

Posttraumatische Belastungsstörung · Epidemiologie - Traumatische Ereignisse $\cdot 2$. Weltkrieg · Höheres Lebensalter

\section{Post-traumatic stress disorder in Germany. Results of a nationwide epidemiological study}

\begin{abstract}
In a representative epidemiological study $(n=2426)$ with a broad age range of respondents (14-93 years), prevalence rates of traumatic life events, post-traumatic stress disorder (PTSD), and partial PTSD were estimated. A standardized interview using the trauma checklist of the Composite International Diagnostic Interview and a DSM-IV PTSD symptom checklist (Modified PTSD Symptom Scale) were applied. One-month prevalence rates were $2.3 \%$ for DSM-IV PTSD and $2.7 \%$ for partial PTSD. There were no gender differences but age-group differences did appear: among persons older than 60 , the prevalence of PTSD was $3.4 \%$, whereas the prevalence was estimated at $1.3 \%$ among persons aged
\end{abstract}

$14-29$ years and $1.9 \%$ among those aged 30 59 years. Partial PTSD exhibited the same age distribution, with $3.8 \%$ in the elderly, $2.4 \%$ in the middle-aged, and $1.3 \%$ in young adults. The results correspond with those of other international studies taking war-related consequences for older age groups into account. Our representative study provides the first evidence of higher PTSD prevalence rates among older age groups in the German population, which is assumed to be related to consequences of World War II.

\section{Keywords}

Post-traumatic stress disorder - Epidemiology · Traumatic events · World War II · Elderly
Beeinträchtigungskriterium hinsichtlich der vor dem Trauma üblichen Lebensführung) wurde beibehalten.

Die PSS zeigte in amerikanischen [14] und deutschen Studien [16] sehr befriedigende Reliabilitäts- und Validitätswerte. Die verkürzte Itemauswahl nach Breslau et al. [15], die sich nach einer Receiver Operating Characteristic Analysis als effektivste Itemauswahl ergeben hatte, definierte positive PTBS-Fälle im Vergleich zur vollständigen Symptomkriterienliste mit einer Sensitivität von $80 \%$, einer Spezifität von $97 \%$, einem positiven prädiktiven Wert von $71 \%$ und einem negativen prädiktiven Wert von 98\% [15].

\section{Diagnose - Vollbild und partielle PTBS}

Das PTBS-Vollbild wurde in Übereinstimmung mit den DSM-IV-Kriterien bestimmt, indem der Algorithmus der modifizierten PTSD-Symptomskala nach Breslau angewendet wurde (Vorliegen des A1- und A2-Kriteriums, B-Kriterien mindestens 4 von 7 Symptomen nach Breslau mit Skalenwerten $\geq 2$ sowie F-Kriterium).

$\mathrm{Da}$ sich in epidemiologischen und in klinischen Studien die Miterfassung der partiellen (subsyndromalen) Störungsbilder eingebürgert hat [17] und die DSMIV-Kriterien eine engere Definition als die weitergefasste ICD-10-Beschreibung der PTBS beinhalten [18], wurden in der vorliegenden Surveystudie auch die weiter gefassten Kriterien für partielle PTBS erhoben. Insbesondere wurde in der letzten Zeit das klinische Beeinträchtigungskriterium untersucht, da dessen Status für die aus PTBS stammenden psychischen Belastungen noch unklar ist [19].

Zwei partielle PTBS-Syndrome wurden spezifiziert:

- Partielle PTBS I: Hier liegen aus den Symptomgruppen B bis D mindestens 2 Symptome vor, zugleich muss aber das F-Kriterium (Beeinträchtigungskriterium im DSM-IV) erfüllt sein (vgl. [17]).

- Partielle PTBS II: Wie bei der partiellen PTBS I liegen mindestens 2 Symptome vor, allerdings ist das FKriterium (Beeinträchtigungskriterium im DSM-IV) nicht angegeben worden (vgl. [19]). 
Hier steht eine Anzeige.

算 Springer 


\begin{tabular}{|c|c|c|c|c|c|c|c|c|c|c|c|c|c|c|}
\hline \multirow[t]{2}{*}{ Trauma } & \multicolumn{2}{|c|}{$\begin{array}{l}\text { Gesamt } \\
(n=2426)\end{array}$} & \multicolumn{2}{|c|}{$\begin{array}{l}\text { Frauen } \\
(n=1308)\end{array}$} & \multicolumn{2}{|c|}{$\begin{array}{l}\text { Männer } \\
(n=1118)\end{array}$} & \multirow{2}{*}{$\begin{array}{l}\text { Test } \\
\text { für Ge- } \\
\text { schlecht } \\
{\left[x^{2}\right]}\end{array}$} & \multicolumn{2}{|c|}{$\begin{array}{l}\text { 14- bis } \\
\text { 29-Jährige } \\
(n=382)\end{array}$} & \multicolumn{2}{|c|}{$\begin{array}{l}30 \text { - bis } \\
59-J a ̈ h r i g e \\
(n=1230)\end{array}$} & \multicolumn{2}{|c|}{$\begin{array}{l}\text { 60- bis } \\
93-J a ̈ h r i g e \\
(n=814)\end{array}$} & \multirow{2}{*}{$\begin{array}{l}\text { Test für } \\
\text { Alters- } \\
\text { gruppen } \\
{\left[x^{2}\right]}\end{array}$} \\
\hline & {$[\mathrm{n}]$} & [\%] & [n] & {$[\%]$} & [n] & [\%] & & {$[\mathrm{n}]$} & [\%] & {$[n]$} & [\%] & {$[n]$} & [\%] & \\
\hline \multicolumn{15}{|l|}{ Kriegsbezogene Traumen } \\
\hline Kriegshandlungen (direkt) & 197 & 8,16 & 111 & 8,52 & 86 & 7,73 & 0,493 & 0 & 0 & 5 & 0,41 & 192 & 23,67 & $392,5^{* * *}$ \\
\hline Ausgebombt im Krieg & 170 & 7,04 & 93 & 7,14 & 77 & 6,92 & 0,046 & 0 & 0 & 3 & 0,24 & 167 & 20,62 & $343,4^{* * *}$ \\
\hline Heimatvertrieben & 161 & 6,66 & 90 & 6,90 & 71 & 6,37 & 0,27 & 3 & 0,79 & 12 & 0,98 & 146 & 17,98 & $252,2^{* * *}$ \\
\hline Gefangenschaft/Geiselnahme & 38 & 1,57 & 6 & 0,46 & 32 & 2,88 & $22,66^{* * *}$ & 0 & 0 & 2 & 0,16 & 36 & 4,44 & $64,96^{* * *}$ \\
\hline \multicolumn{15}{|l|}{ Zivile Traumen } \\
\hline Vergewaltigung & 18 & 0,75 & 16 & 1,23 & 2 & 0,18 & $8,91^{* *}$ & 4 & 1,05 & 6 & 0,49 & 8 & 0,99 & 2,21 \\
\hline Kindesmissbrauch (<14. Lebensjahr) & 29 & 1,20 & 24 & 1,84 & 5 & 0,45 & $9,84^{* *}$ & 5 & 1,32 & 17 & 1,39 & 7 & 0,87 & 1,16 \\
\hline Schwerer Unfall & 111 & 4,59 & 40 & 3,07 & 71 & 6,38 & $15,10^{* * *}$ & 10 & 2,63 & 55 & 4,49 & 46 & 5,67 & 5,52 \\
\hline Körperliche Gewalt & 91 & 3,77 & 32 & 2,46 & 59 & 5,30 & $13,35^{* * *}$ & 6 & 1,58 & 16 & 1,31 & 69 & 8,53 & $76,04^{* * * *}$ \\
\hline Lebensbedrohliche Krankheit & 72 & 2,98 & 36 & 2,76 & 36 & 3,23 & 0,47 & 2 & 0,53 & 25 & 2,04 & 45 & 5,56 & $30,28^{* * * *}$ \\
\hline Naturkatastrophe & 19 & 0,79 & 9 & 0,69 & 10 & 0,90 & 0,34 & 1 & 0,26 & 5 & 0,41 & 13 & 1,61 & $10,56^{* *}$ \\
\hline Zeuge eines Traumas & 204 & 8,45 & 112 & 8,61 & 92 & 8,27 & 0,087 & 14 & 3,68 & 62 & 5,06 & 128 & 15,86 & $86,66^{* * *}$ \\
\hline Andere & 87 & 3,61 & 54 & 4,16 & 33 & 2,98 & 2,38 & 6 & 1,58 & 34 & 2,78 & 47 & 5,85 & $18,45^{* * *}$ \\
\hline
\end{tabular}

aMehrfachangaben möglich.

Tab. 3 Einmonatsprävalenz der PTBS und partieller PTBS-Syndrome im Geschlechts- und Altersgruppenvergleich

\begin{tabular}{|c|c|c|c|c|c|c|c|c|c|c|c|c|c|c|}
\hline \multirow[t]{2}{*}{ Syndrome } & \multicolumn{2}{|c|}{$\begin{array}{l}\text { Gesamt } \\
(n=2426)\end{array}$} & \multicolumn{2}{|c|}{$\begin{array}{l}\text { Frauen } \\
(n=1308)\end{array}$} & \multicolumn{2}{|c|}{$\begin{array}{l}\text { Männer } \\
(n=1118)\end{array}$} & \multirow{2}{*}{$\begin{array}{l}\text { Test } \\
\text { für Ge- } \\
\text { schlecht } \\
{\left[x^{2}\right]}\end{array}$} & \multicolumn{2}{|c|}{$\begin{array}{l}\text { 14- bis } \\
\text { 29-Jährige } \\
(n=382)\end{array}$} & \multicolumn{2}{|c|}{$\begin{array}{l}\text { 30- bis } \\
\text { 59-Jährige } \\
(n=1230)\end{array}$} & \multicolumn{2}{|c|}{$\begin{array}{l}\text { 60- bis } \\
93-J a ̈ h r i g e \\
(n=814)\end{array}$} & \multirow{2}{*}{$\begin{array}{l}\text { Test für } \\
\text { Alters- } \\
\text { gruppen } \\
{\left[x^{2}\right]}\end{array}$} \\
\hline & [n] & [\%] & [n] & [\%] & [n] & [\%] & & [n] & [\%] & [n] & [\%] & [n] & [\%] & \\
\hline 1. PTBS (Vollbild) & 56 & 2,31 & 33 & 2,52 & 23 & 2,06 & 0,580 & 5 & 1,31 & 23 & 1,87 & 28 & 3,44 & $7,362^{*}$ \\
\hline $\begin{array}{l}\text { 2. Partielle PTBSI } \\
\text { (mit Beeinträchtigungskriterium) }\end{array}$ & 18 & 0,74 & 8 & 0,61 & 10 & 0,89 & 0,655 & 2 & 0,52 & 8 & 0,65 & 8 & 0,98 & 1,029 \\
\hline $\begin{array}{l}\text { 3. Partielle PTBS II } \\
\text { (ohne Beeinträchtigungskriterium) }\end{array}$ & 48 & 1,98 & 26 & 1,99 & 22 & 1,97 & 0,001 & 3 & 0,79 & 22 & 1,79 & 23 & 2,83 & $6,04^{*}$ \\
\hline Summe & 122 & 5,03 & 67 & 5,12 & 5 & 4,92 & 0,052 & 10 & 2,62 & 53 & 4,31 & 59 & 7,25 & $14,38^{* * * *}$ \\
\hline
\end{tabular}

\section{Ergebnisse}

\section{Häufigkeit traumatischer Ereignisse}

Achtundzwanzig Prozent $(\mathrm{n}=314)$ der Frauen und 20,9\% $(n=274)$ der Männer berichteten mindestens ein traumatisches Ereignis (Geschlechtsunterschied $\chi^{2}=0,08$; n.s.). Bezogen auf die Altersgruppen waren es $9,9 \%(n=38)$ der jungen Erwachsenen, $13,3 \%(n=164)$ der mittelalten Erwachsenen sowie $47,4 \%(n=386)$ der über 6o-Jährigen, die mindestens ein traumatisches Ereignis berichteten $\left(\chi^{2}=360,36\right.$; $\mathrm{p}<\mathrm{O}, \mathrm{OO} 1)$.

Im Einzelnen ergab sich folgende Verteilung von Mehrfachtraumatisierungen: $12 \%$ aller Untersuchten $(n=292)$ gaben nur ein Trauma an, 5,6\% $(n=135)$ berichteten 2 Traumata, 2,9\% $(n=71)$ gaben 3 Traumata an, 2,0\% $(n=49)$ berichteten 4 Traumata und $1,7 \%(\mathrm{n}=49)$ gaben 5 oder mehr Traumata an. Zwischen den Geschlech- tern unterscheiden sich die Häufigkeiten der Mehrfachtraumatisierungen nicht $\left(\chi^{2}=0,33\right.$, n.s. $)$.

Für die Auswertung der traumatischen Ereignisse wurden 2 Kategorien gebildet: kriegsbezogene und zivile Traumen (- Tab. 2). Von den kriegsbezogenen Traumen waren in der Gruppe der Älteren die direkten Kriegserlebnisse mit 23,7\% am häufigsten, gefolgt vom Ausgebombtsein im Krieg $(20,6 \%)$ und traumatischen Ereignissen im Zusammenhang der Heimatvertreibung $(17,9 \%)$.

Die zivilen Traumen waren ebenfalls in der Gruppe der Älteren am häufigsten (dort gaben 15,7\% an, Zeuge eines Traumas gewesen zu sein, gefolgt von $8,5 \%$ mit erlebter körperlicher Gewalt). In der Gruppe der Mittelalten bzw. der Jüngeren waren ebenfalls die Traumazeugenschaft (5,1\% bzw. 3,7\%) sowie schwere Unfälle ( $4,5 \%$ bzw. $2,6 \%$ ) am häufigsten.
Für 4 Ereignisgruppen liegen Geschlechtsunterschiede vor:

- bei Vergewaltigung und Kindesmissbrauch mit signifikant höheren Häufigkeiten für Frauen sowie - für schwere Unfälle und körperliche Gewalt mit signifikant höheren Häufigkeiten für Männer.

\section{Einmonatsprävalenzraten der PTBS}

Insgesamt liegt das Vollbild der PTBS bei $2,3 \%$ der Befragten vor, wobei 2,5\% der Frauen und 2,1\% der Männer betroffen sind (• Tab. 3). Im Altersgruppenvergleich ergibt sich ein signifikanter Anstieg über die Altersgruppen mit 1,3\% bei den Jungen, $1,9 \%$ bei den Mittelalten und 3,4\% bei den Älteren. Daraus ergibt sich ein 2,6fach höheres relatives Risiko ( $\mathrm{OR}=2,7$, 95\%CI: 1,03-7,01) für die über 6o-Jährigen gegenüber den jüngeren Erwachsenen, ein Vollbild einer PTBS zu haben. 


\section{Hamburger Fellowship Persönlichkeitsstörungen 2008 ausgeschrieben}

Die Gesellschaft zur Erforschung und Therapie von Persönlichkeitsstörungen (GePs) e.V. und die Asklepios Kliniken Hamburg GmbH schreiben aus: Das Hamburger Fellowship Persönlichkeitsstörungen 2008. Das Fellowship ist mit 5.000 EUR dotiert. Gefördert werden Nachwuchswissenschaftlerinnen und Nachwuchswissenschaftler, die das 40 . Lebensjahr noch nicht vollendet haben und nicht habilitiert sind. Eingereicht werden kann nicht mehr als ein Abstract (keine Manuskripte oder Sonderdrucke) von einer Ori- ginalarbeiten aus der klinischen Forschung zu Persönlichkeitsstörungen, die noch nicht oder nicht vor 2007 veröffentlicht wurde. Der Einreicher muss Erst- oder Seniorautor sein, die Zustimmung aller Co-Autoren zur Einreichung ist vorzulegen; die Arbeit soll im deutschen Sprachraum entstanden sein. Das Fellowship wird zweckgebunden für den Besuch einer internationalen, im Bereich der Persönlichkeitsstörungen bedeutenden wissenschaftlichen oder klinischen Einrichtung vergeben. Bewerber werden gebeten, das Abstract ihrer Arbeit in 5 Exemplaren mit einem kurzen CV beim Präsidenten der Gesellschaft zur Erforschung und Therapie von Persönlichkeitsstörungen (GePs) e.V., Dr. Birger Dulz, Asklepios Klinikum Nord, Langen- horner Chaussee 560, D-22419 Hamburg, bis spätestens zum 30.04.2008 einzureichen. Die Entscheidung über die Vergabe des Preises erfolgt unter Ausschluss des Rechtsweges durch eine Jury, die sich aus Mitgliedern der GePs und fachkundigen Mitarbeitern der Asklepios Kliniken Hamburg GmbH zusammensetzt. Aus den Bewerbern werden von der Jury 4 bis 6 ausgewählt und eingeladen, ihre Arbeiten beim Hamburger Symposium Persönlichkeitsstörungen am 05./06.09.2008 vorzustellen. Von der Jury wird die beste Präsentation ausgewählt und prämiert.

Quelle: Gesellschaft zur Erforschung und Therapie von Persönlichkeitsstörungen (GePs), www.geps.info

\section{Hier steht eine Anzeige.}

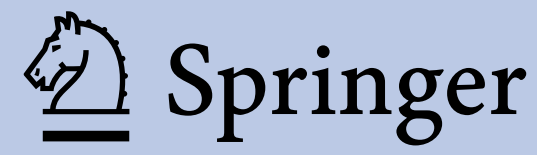




\begin{tabular}{|c|c|c|c|c|c|c|c|c|}
\hline \multirow[t]{2}{*}{ Trauma } & \multicolumn{2}{|c|}{$\begin{array}{l}\text { Subjektiv } \\
\text { schlimms- } \\
\text { tes Trauma }\end{array}$} & \multicolumn{3}{|c|}{$\begin{array}{l}\text { Bedingtes Auftreten der } \\
\text { PTBS-Vollbild }\end{array}$} & \multicolumn{3}{|c|}{$\begin{array}{l}\text { Bedingtes Auftreten der } \\
\text { partiellen PTBS I+II }\end{array}$} \\
\hline & [n] & {$[\%]^{b}$} & [n] & {$[\%]^{\mathrm{c}}$} & $95 \% \mathrm{Cl}$ & [n] & {$[\%]^{c}$} & $95 \% \mathrm{Cl}$ \\
\hline \multicolumn{9}{|l|}{ Kriegsbezogene Traumen } \\
\hline Kriegseinsatz & 89 & 15,14 & 7 & 7,87 & $2,28-13,46$ & 7 & 7,87 & $2,28-13,46$ \\
\hline Ausgebombt im Krieg & 73 & 12,41 & 2 & 2,74 & $0-6,48$ & 1 & 1,37 & $0-4,04$ \\
\hline Heimatvertrieben & 76 & 12,93 & 4 & 5,26 & $0,24-10,28$ & 1 & 1,32 & $0-3,89$ \\
\hline $\begin{array}{l}\text { Gefangenschaft/ } \\
\text { Geiselnahme }\end{array}$ & 7 & 1,19 & 0 & - & - & 2 & 28,57 & $0-62,04$ \\
\hline \multicolumn{9}{|l|}{ Zivile Traumen } \\
\hline Vergewaltigung & 8 & 1,36 & 3 & 37,50 & $3,95-71,05$ & 0 & - & - \\
\hline $\begin{array}{l}\text { Kindesmissbrauch } \\
(<14 . \text { Lebensjahr) }\end{array}$ & 17 & 2,89 & 6 & 35,29 & $12,57-58,01$ & 1 & 5,88 & 17,06 \\
\hline Schwerer Unfall & 78 & 13,27 & 10 & 12,82 & $5,40-20,24$ & 19 & 24,36 & $14,83-33,89$ \\
\hline Körperliche Gewalt & 19 & 3,23 & 2 & 10,53 & $0-24,33$ & 3 & 15,79 & $0-32,19$ \\
\hline $\begin{array}{l}\text { Lebensbedrohliche } \\
\text { Krankheit }\end{array}$ & 47 & 7,99 & 11 & 23,40 & $11,30-35,50$ & 9 & 19,15 & $7,9-30,4$ \\
\hline Naturkatastrophe & 6 & 1,02 & 0 & - & - & 1 & 16,67 & $0-46,49$ \\
\hline Zeuge eines Traumas & 87 & 14,80 & 6 & 6,90 & $1,57-12,23$ & 10 & 11,49 & $4,79-18,19$ \\
\hline Andere Traumata & 33 & 5,61 & 4 & 12,12 & $0,98-23,26$ & 9 & 27,27 & $12,08-42,46$ \\
\hline Mehrere Traumata ${ }^{a}$ & 48 & 8,16 & 1 & 2,08 & $0-6,12$ & 3 & 6,25 & $0-13,1$ \\
\hline
\end{tabular}

aEs wurden mehrere Traumen aber kein „schlimmstes" angegeben; ${ }^{b}$ von $n=588$ mit irgendeinem Trauma; ' ${ }^{c}$ on der jeweiligen Kategorie „subjektiv schlimmstes Trauma".

Die partielle PTBS I, bei der nur einige Symptome der DSM-IV-Symptomgruppen $B, C$ und $D$ vorhanden sind und das Beeinträchtigungskriterium $\mathrm{F}$ erfüllt ist, liegt hinsichtlich der Einmonatsprävalenzrate bei $0,7 \%$ der Befragten vor. Die partielle PTBS I weist ebenfalls einen signifikanten Altersanstieg auf (• Tab. 3) mit einem nicht signifikanten 1,9fach höheren relativen Risiko (OR=1,9, 95\%CI: 0,40$8,92)$ der Älteren gegenüber den Jüngeren.

Die partielle PTBS II (einige Symptome ohne subjektives Beeinträchtigungskriterium) weist eine Einmonatsprävalenzrate von 2,0\% auf. Wiederum existiert kein Geschlechtsunterschied, aber ein signifikanter Altersanstieg. Das relative Risiko für ein partielles PTBS-Syndrom liegt für die Älteren um 3,6fach (OR=3,0, 95\%CI: 1,15-7,74) höher als für die Jüngeren.

Für die Summe aller 3 PTBS-Syndrome zeigt $\bullet$ Tab. 3 die Ergebnisse. Aufgrund der höheren Fallzahlen wurden für diese zusammengefassten Prävalenzen die $\mathrm{Zu}$ sammenhänge mit den weiteren soziodemographischen Variablen (Partnerschaft, Bildung, Erwerbstätigkeit, Wohnsitz) berechnet, die aber alle nicht signifikant waren.

\section{Bedingte Wahrscheinlichkeiten einer aktuellen PTBS}

Die gemittelte bedingte Wahrscheinlichkeit, eine aktuelle PTBS bei einem im Lebenszeitraum vorliegenden traumatischen Erlebnis auszubilden, war 12,0\% für das Vollbild und zusätzliche $12,8 \%$ für die partiellen PTBS-Syndrome. Als traumatische Lebenserfahrungen mit der größten Wahrscheinlichkeit einer PTBS-Entwicklung erwiesen sich - in absteigender Reihenfolge - Vergewaltigungen, Kindesmissbrauch und lebensbedrohliche Erkrankungen (PTBS-Vollbilder: 37,5\%, 35,3\% bzw. 23,4\%; - Tab. 4). Die kriegsbedingten Traumata zeigten insgesamt geringere bedingte Wahrscheinlichkeiten für eine PTBS zum Untersuchungszeitpunkt (unter 8\%).

Die zusammengefassten partiellen PTBS-Wahrscheinlichkeiten waren dagegen teilweise bei anderen Traumakategorien höher. Nach der Traumakategorie „schwere Unfallle" mit 24,36\%, waren es die „lebensbedrohlichen Erkrankungen" mit 19,15\% und die körperliche Gewalt mit 15,79\%, die vergleichsweise häufig mit einer aktuellen partiellen PTBS in Verbindung stehen.

\section{Diskussion}

In dieser Studie wurde erstmals eine große repräsentative Bevölkerungsstichprobe in Deutschland anhand gut validierter Screeninginstrumente auf das Vorliegen der posttraumatischen Belastungsstörung nach DSM-IV sowie partieller Störungsbilder untersucht. Der Altersbereich war umfassender als in vergleichbaren internationalen Studien und reichte bis über das 9o. Lebensjahr.

Hauptergebnisse sind zunächst die niedrigeren Traumaereignis- und PTBSPrävalenzraten im Vergleich zu den viel zitierten älteren US-amerikanischen Studien von Kessler et al. [1] und Breslau [2]. Ein Viertel der Befragten berichtete traumatische Lebensereignisse und 2,3\% eine aktuelle Einmonatsprävalenz des Vollbilds der PTBS. Dazu kommen noch 2,7\% partielle PTBS-Syndrome.

In Bezug auf die geringeren berichteten Häufigkeiten von traumatischen Ereignissen ist auf die etwas ältere Form der eingesetzten Traumaliste hinzuweisen. Sie hat in den vergangenen $15 \mathrm{Jah}-$ ren verschiedene Überarbeitungen hinsichtlich Erweiterungen gefunden [20] und scheint in dieser älteren Form einen relativ großen Bereich derjenigen traumatischen Erfahrungen, der im Zusammenhang mit Traumatisierungen durch schreckliche Ereignisse bei nahestehenden anderen Personen steht, nicht abzudecken und ist daher relativ restriktiv hinsichtlich der Definition von Traumata. Auch in den USA wurde erst im Verlauf verschiedener Untersuchungen in diesem Zeitraum auf die Einbeziehung dieser Kategorie hingewiesen [21], die in neueren Studien teilweise den größten Anteil an der Anzahl traumatischer Ereignistypen hat. Eine diesbezügliche Bestätigung der restriktiven Definition traumatischer Ereignisse findet sich auch durch die relativ hohen bedingten Wahrscheinlichkeiten für eine volle PTBS-Störungen bei der gegenwärtigen Untersuchung. Diese Restriktion hinsichtlich der Definition von psychischen Traumata ist jedoch im Weiteren zu beachten.

Die vergleichsweise niedrige Prävalenz des PTBS-Vollbildes lassen sich durch mehrere Gründe erklären: 
1. fanden viele der vorliegenden Studien (z. B. in verschiedenen europäischen Staaten, Australien und Mexiko) ebenfalls niedrigere Prävalenzraten als die aus den USA berichteten $[6,7,8,9]$;

2. wurden hier nur Einmonatsprävalenzraten geschätzt und nicht wie bei Kessler, Breslau und Kollegen Lebenszeitprävalenzraten. Lebenszeitprävalenzraten sind naturgemäß höher als Punktprävalenzraten, da sie auch die ausgeheilten Fälle umfassen.

Ein weiteres Hauptergebnis ist der fortschreitende Anstieg von PTBS und partieller PTBS über die Altersgruppen. Dabei fanden sich bei den über 6o-Jährigen gegenüber den jüngeren Altersgruppen 2- bis 3 fach erhöhte Prävalenzraten für PTBS. Dieser Befund ist unseres Wissens neu in der internationalen Literatur, da bisher von erhöhten Inzidenzraten vor allem während der Zeit der Adoleszenz und dem jüngeren Erwachsenenalter ausgegangen wurde [21]. Die relativ hohen Raten bei Älteren können mit den Kriegserfahrungen des 2. Weltkriegs der deutschen Bevölkerung in Zusammenhang gebracht werden $[22,23,24,25,26]$. Diese Kriegserfahrungen müssen nicht in direktem Zusammenhang mit aktuellen PTBS-Symptomen stehen, können aber das Risiko für das Auftreten einer neuen Symptomatik nach einem weiteren Trauma erhöhen. Verschiedene Studien haben auf den Risikofaktor der multiplen Traumatisierungen für neue Symptommanifestationen hingewiesen (z. B. [12]). Andererseits können die durch diese Kriegserfahrungen hervorgerufenen PTSD-Symptome auch durch spätere Traumatisierungen aufrechterhalten werden [20]. Die Annahme der Einwirkung von Kriegshandlungen wird indirekt auch gestützt durch die repräsentative Studie in Portugal, deren vergleichsweise höheren PTBSPrävalenzen durch die Kolonial- und Bürgerkriege des Landes in den 1960er und 1970er Jahren begründet wurden [9]. In einer eigenen Studie in der Schweiz [27] fanden sich dagegen bei Älteren (6595 Jahre) nur bei o,7\% das PTBS-Vollbild und bei $4,2 \%$ ein subsyndromales PTBS, was ebenfalls für die - in diesem Fall fehlende direkte - Kriegseinwirkung auf die
PTBS-Prävalenzen spricht. Diese Ergebnisse könnten darauf hinweisen, dass das direkte Erleben von Kriegsereignissen in Form von Kriegshandlungen, Verlust des Zuhauses durch Bombardierung, Vertreibung und Gefangenschaft selbst nach mehr als 6o Jahren einen negativen Effekt auf das psychische Wohlbefinden hat. Hingegen sind Menschen, die kurz nach dem Krieg geboren wurden, das heißt durch die Kriegsauswirkungen durchaus ebenfalls noch belastet waren, deutlich weniger dem Risiko ausgesetzt, an einer PTBS zu erkranken.

Die Studie untersuchte neben dem PTBS-Vollbild nach der DSM-IV-Definition auch zwei partielle PTBS-Syndrome, wie sie in der Literatur diskutiert werden $[17,19]$. Generell waren die partiellen PTBS-Prävalenzen ein bis eineinhalb Mal so hoch wie die des PTBS-Vollbildes. Dies spricht dafür, dass in klinischen und epidemiologischen Studien die partiellen PTBS-Bilder wie bisher berücksichtigt werden sollten, da es durch die Absenkung der diagnostischen Schwelle nicht zu einer exzessiven Zunahme der Prävalenzschätzungen kommt. Schützwohl und Maercker [17] haben ebenso wie Stein et al. [28] gezeigt, dass die Personen mit partieller PTBS ebenso wie Personen mit dem PTBS-Vollbild sowohl erhöhte psychopathologische Belastungswerte als auch Alltagseinschränkungen haben. Dem trägt die klinische Praxis in Europa auch Rechnung, indem sie die Diagnosekriterien des ICD-10 regulär einsetzt, die liberaler sind als die des DSM-IV [18].

Eine weitere Interpretation der Ergebnisse sollte vor dem Hintergrund der Stärken und Schwächen der vorliegenden Studie erfolgen. Stärken bestehen in der erstmaligen Untersuchung einer Bevölkerungsstichprobe einschließlich Älterer in Deutschland. Eine Schwäche der Studie hinsichtlich der restriktiven Definition traumatischer Ereignisse wurde bereits angesprochen. Ein Schwachpunkt der Studiendurchführung, der auch hinsichtlich der Häufigkeit von Traumataberichten relevant ist, bezieht sich auf die Methodik der zum Teil in Fragebogenform vorgelegten Fragen durch nichtmedizinische/-psychologische Interviewer eines Umfrageinstituts. Damit verbunden könnte eine unsichere Compli- ance der Befragten in Bezug auf Auskünfte über ihre psychischen Probleme vorliegen. Daher könnten die vorliegenden Daten zu einer weiteren Unterschätzung führen, die sich z. B. zusätzlich bei den berichteten traumatischen Erfahrungen auswirkt. Die meisten der hier diskutierten anderen Repräsentativstudien sind zwar ebenfalls durch Interviews mit geschulten Laien erhoben worden, verwendeten aber standardisierte klinische Interviews zur Erfassung der PTBS-Problematik. Des Weiteren ist bei Untersuchungen dieser Art immer eine Erinnerungsverzerrung bzw. eine Gedächtnisproblematik in Betracht zu ziehen, wenn weit zurückliegende Ereignisse erfasst werden sollen. Dem kann jedoch bei der vorliegenden Untersuchung hinsichtlich der Folgen der Ereignisse entgegengestellt werden, dass die Schätzungen der PTBS-Symptomatik für Punktprävalenzraten relativ hoch sind und sich nur auf die aktuelle Symptomatik beziehen. Die ausschließliche Erhebung und Schätzung von Punktprävalenzraten der Störungen kann allerdings auch als eine Schwäche der Studie diskutiert werden, da die meisten Untersuchungen Angaben zu Lebenszeitprävalenzraten beinhalten und die Vergleichbarkeit somit eingeschränkt ist. Hier muss auf die eingangs beschriebene Beschränkung der Untersuchungsplanung hingewiesen werden, in der es nur möglich war, einen vergleichsweise geringen Datenumfang in einer gesamtdeutschen, durch ein Umfrageinstitut vorgenommenen Studie zu erfragen.

Trotz dieser Restriktionen zeigt die Studie aber, dass in den USA erhobene Repräsentativdaten zur PTBS wie die aus der National Comorbidity Survey [1] nur mit Vorbehalt zur Grundlage von Prävalenzschätzungen in Deutschland herangezogen werden können.

\section{Fazit für die Praxis}

Die von uns gefundenen Traumatisierungs- und die PTBS-Raten sind erfreulicherweise etwas geringer als in den USA. Die relativ hohen Raten bei Personen im höheren Lebensalter, für die wir insbesondere kriegsbedingte Faktoren annehmen, sind ein wichtiges Ergebnis, das nur in einer Bevölkerungsstichprobe wie der vorliegenden oder aus einem ande- 
ren Land mit ganz direkten Kriegseinwirkungen beobachtet werden kann. Der Altersgipfel der PTBS in Deutschland hat wichtige psychiatrisch-psychotherapeutische Konsequenzen, so dass bei der Diagnostik und Differenzialdiagnostik älterer Menschen die posttraumatische Belastungsstörung in Erwägung gezogen werden sollte. Psychotherapeutische und pharmakotherapeutische Möglichkeiten sollten geprüft werden, wobei hier altersadaptierte Verfahren bzw. Dosierungen eingesetzt werden sollten. Für Patienten aller Lebensalter gilt darüber hinaus, dass traumatisierende Lebensereignisse zu einer PTBS führen können, wobei die Folgen sexualisierter Gewalt (Kindesmissbrauch, Vergewaltigung) die vergleichsweise höchste Pathogenität besitzen.

\section{Korrespondenzadresse}

\section{Prof. Dr. Dr. A. Maercker}

Fachrichtung Psychopathologie und Klinische Intervention, Universität Zürich Binzmühlestrasse 14/17, 8050 Zürich Schweiz maercker@psychologie.unizh.ch

Interessenkonflikt. Der korrespondierende Autor gibt an, dass kein Interessenkonflikt besteht.

\section{Literatur}

1. Kessler RC, Sonnega A, Bromet A et al. (1995) Posttraumatic stress disorder in the National Comorbidity Survey. Arch Gen Psychiatry 52: 1048-1060

2. Breslau N (2002) Epidemiologic studies of trauma, posttraumatic stress disorder, and other psychiatric disorders. Can J Psychiatry 47: 923-929

3. Perkonigg A, Pfister H, Stein MB et al. (2000) Longitudinal course of post traumatic stress disorder and post traumatic stress disorder symptoms in a community of adolescents and young adults. Am J Psychiatry 162: 1320-1327

4. Maercker A, Michael T, Fehm L et al. (2004) Age of traumatisation as a predictor of PTSD or major depression in young women. Br J Psychiatry 184: 482-487

5. Hapke U, Schumann A, Rumpf H-J et al. (2006) Post-traumatic stress disorder: the role of trauma, pre-existing psychiatric disorders, and gender. Eur Arch Psychiatry Clin Neurosci 256: 299-306

6. Alonso J, Angermeyer MC, Bernert S et al. (2004) Prevalence of mental disorders in Europe: results from the European Study of the Epidemiology of Mental Disorders (ESEMeD) project. Acta Psychiatr Scand 109: 21-27

7. Medina-Morca Icaza ME, Borges-Guimaraes G, Lara $C$ et al. (2005) Prevalence of violent events and post-traumatic stress disorder in the Mexican population. Salud Publica Mex 47: 8-22

8. Creamer M, Burgess P, McFarlane AC (2001) Posttraumatic stress disorder: findings from the Australian national survey of mental health and wellbeing. Psychol Med 32: 1237-1247
9. DeAlbuquerque A, Soares C, De Jesus PM, Alves C (2003) Post-traumatic stress disorder. Assessment of its rate of occurrence in the adult population of Portugal. Acta Med Portugal 16: 309-320

10. Maercker A (Hrsg) (2003) Erscheinungsbild, Erklärungsansätze und Therapieforschung. Therapie der posttraumatischen Belastungsstörungen. 2 Aufl. Springer, Berlin, S 3-36

11. Statistisches Bundesamt (Hrsg) (2006) Statistisches Jahrbuch 2006 für die Bundesrepublik Deutschland. Eigendruck, Wiesbaden

12. Perkonigg A, Kessler RC, Storz S, Wittchen HU (2000) Traumatic events and post-traumatic stress disorder in the community: prevalence, risk factors and comorbidity. Acta Psychiatr Scand 101: 46-59

13. Wittchen H-U, Pfister H (Hrsg) (1997) DIA-X-Interviews: Manual für Screening-Verfahren und Interview, Interviewheft. Swets \& Zeitlinger, Frankfurt

14. Foa EB, Riggs DS, Dancu CV, Rothbaum BO (1993) Reliability and validity of a brief instrument for assessing post-traumatic stress disorder. J Trauma Stress 6: 459-473

15. Breslau N, Peterson EL, Kessler RC, Schultz LR (1999) Short screening scale for DSM-IV posttraumatic stress disorder. Am J Psychiatry 156: 908911

16. Stieglitz RD, Frommberger U, Foa EB, Berger M (2001) Evaluation of the German version of the PTSD Symptom Scale (PSS). Psychopathology 34: 128-133

17. Schützwohl M, Maercker A (1999). Effects of varying diagnostic criteria for PTSD. J Trauma Stress 12 155-165

18. Rosner R, Powell S (2007) Überschätzt die ICD-10 die PTBS-Prävalenz? Trauma Gewalt 1: 46-61

19. Breslau N, Alvarado GF (2008) The clinical significance criterion in DSM-IV post-traumatic stress disorder. Psychol Med 20: 1-8

20. Perkonigg A, Pfister $\mathrm{H}$, Lieb R, Wittchen $\mathrm{H}-\mathrm{U}$ (2005) Traumatic events and PTSD among adolescents and youg adults in Germany: prevalence and specific characteristics in a representative commuity sample. Vortrag gehalten in Toronto beim 22nd Annual Meeting der International Society of Traumatic Stress Studies

21. Breslau N (2001) The epidemiology of posttraumatic stress disorder: What is the extent of the problem? J Clin Psychiatry [Suppl 17] 62: 16-22

22. Brähler E, Gunzelmann T, Glaemer H, Maercker A (2006) Posttraumatische Belastungsstörungen bei deutschen Seniorinnen und Senioren - Ergebnisse einer Repräsentativbefragung. Manuskript

23. Maercker A, Herrle J, Grimm I (1999) Dresdener Bombennachtsopfer 50 Jahre danach. Eine Untersuchung patho- und salutogenetischer Variablen. Z Gerontopsych Psychiatr 3: 157-167

24. Radebold H (2005) Zeitgeschichtliche Erfahrungen und ihre Folgen-notwendige weitere Perspektive bei der Psychotherapie Älterer. Psychotherapie im Alter 4: 75-87

25. Maercker A (Hrsg) (2002) Posttraumatische Belastungsstörungen und komplizierte Trauer. Alterspsychotherapie und klinische Gerontopsychologie. Springer, Berlin, S 245-282

26. Heuft G (1999) Die Bedeutung der Trauma-Reaktivierung im Alter. Z Gerontol Geriatr 32: 225-230

27. Maercker A, Forstmeier S, Enzler A et al. (2008) Adjustment disorders, PTSD and depressive disorders in old age: Findings from a community survey. Compr Psychiatry 49: 113-120

28. Stein MB, Höfler M, Perkonigg A et al. (2002) Patterns of incidence and psychiatric risk factors for traumatic events. Int J Methods Psychiatr Res 11: 143-153

\section{Hormosan Forschungspreis für Psychiatrie 2008}

Die Firma Hormosan Pharma, Frankfurt, stiftete den Hormosan Forschungspreis für Psychiatrie. Der Preis wird jährlich ausgeschrieben und ist mit $10.000 €$ dotiert.

Die Bewerbungsfrist endet am 30. Juni 2008. Nähere Informationen können über den Vorsitzenden des Kuratoriums des Hormosan Forschungspreises für Psychiatrie, Herrn Professor Dr. H.-J. Möller, Psychiatrische Klinik der Ludwig-Maximilians-Universität, Nußbaumstraße 7, 80336 München, erfragt werden.

Quelle: Herr Professor Möller (München). 